\title{
Space weather effects on drilling accuracy in the North Sea
}

\author{
S. J. Reay ${ }^{1}$, W. Allen ${ }^{2}$, O. Baillie ${ }^{1}$, J. Bowe ${ }^{3}$, E. Clarke ${ }^{1}$, V. Lesur ${ }^{1}$, and S. Macmillan ${ }^{1}$ \\ ${ }^{1}$ British Geological Survey, Murchison House, West Mains Road, Edinburgh EH9 3LA, UK \\ ${ }^{2}$ Halliburton (Sperry Drilling Services), 6900 Arctic Boulevard, Anchorage, Alaska, AK 99518, USA \\ ${ }^{3}$ Halliburton (Sperry Drilling Services), Howe Moss Drive, Kirkhill Industrial Estate, Aberdeen, AB21 0GL, UK
}

Received: 22 February 2005 - Revised: 11 August 2005 - Accepted: 20 August 2005 - Published: 22 November 2005

Part of Special Issue "1st European Space Weather Week (ESWW)"

\begin{abstract}
The oil industry uses geomagnetic field information to aid directional drilling operations when drilling for oil and gas offshore. These operations involve continuous monitoring of the azimuth and inclination of the well path to ensure the target is reached and, for safety reasons, to avoid collisions with existing wells. Although the most accurate method of achieving this is through a gyroscopic survey, this can be time consuming and expensive. An alternative method is a magnetic survey, where measurements while drilling (MWD) are made along the well by magnetometers housed in a tool within the drill string. These MWD magnetic surveys require estimates of the Earth's magnetic field at the drilling location to correct the downhole magnetometer readings. The most accurate corrections are obtained if all sources of the Earth's magnetic field are considered. Estimates of the main field generated in the core and the local crustal field can be obtained using mathematical models derived from suitable data sets. In order to quantify the external field, an analysis of UK observatory data from 1983 to 2004 has been carried out. By accounting for the external field, the directional error associated with estimated field values at a mid-latitude oil well $\left(55^{\circ} \mathrm{N}\right)$ in the North Sea is shown to be reduced by the order of $20 \%$. This improvement varies with latitude, local time, season and phase of the geomagnetic activity cycle. By accounting for all sources of the field, using a technique called Interpolation In-Field Referencing (IIFR), directional drillers have access to data from a "virtual" magnetic observatory at the drill site. This leads to an error reduction in positional accuracy that is close to matching that of the gyroscopic survey method and provides a valuable independent technique for quality control purposes.
\end{abstract}

Keywords. Geomagnetism and Paleomagnetism (Instruments and technique; Rapid time variations; Time variations, Diurnal to secular)

Correspondence to: S. J. Reay

(sjr@bgs.ac.uk)

\section{Introduction}

Directional drilling, which involves directing a borehole along a predetermined trajectory to intersect a designated subsurface target, is a technique essential for the exploitation of oil and gas reserves. The trajectory of the borehole is controlled by changing the orientation of the drill bit, making it possible to drill multiple wellbores to different sections of a reservoir from a single structure, which is particularly useful for offshore operations. Measuring the true position of the borehole along its trajectory is critical to ensure the well penetrates its target and avoids collisions with existing wells. The actual well path trajectory is mathematically integrated from a series of discrete survey measurements taken along the length of the borehole. At each survey station the wellbore inclination and direction are measured and assigned to the measured depth of the survey point. Wellbore inclination is defined as the angle from the vertical to the wellbore axis and wellbore direction is the angle in the horizontal plane from a defined north reference to the wellbore axis (often referred to as azimuth). The measured depth is the total (calculated) length along the well path.

There are a number of surveying methods used in directional drilling and in practice often more than one method is used. In general, the most accurate values of wellbore inclination and direction are obtained from measurements made using gyroscopic tools. However, these surveys can be relatively expensive as drilling operations are usually suspended for several hours while the survey is conducted. In addition to this, the most accurate gyroscopic surveys must be run in borehole sections that have been completed and lined with steel casing that has been cemented into position. Consequently, it may be too late to remedy any significant discrepancies between the true wellbore position and the planned trajectory or it may involve expensive correctional drilling to realign the well path. To avoid the risk of missing reservoir targets it is now common practice to run intermediate gyroscopic surveys during the drilling operation. However, this 
comes at the cost of degraded survey quality and an increased risk of technical problems, further delaying the drilling operation.

To resolve the shortcomings of gyroscopic survey tools an alternative survey method uses magnetometers and accelerometers, secured in the drill string to make measurements while drilling (MWD). This saves drilling time and reduces the technical risk, therefore keeping drilling costs to a minimum. Magnetic MWD tools usually comprise three accelerometers measuring the gravity field vector and three fluxgate sensors measuring the Earth's magnetic field vector. The three sensors in each set are orthogonal, and the corresponding magnetic and gravity sensor axes are closely aligned. For the MWD method to be viable, the accuracy of the measured borehole position must be comparable with that achieved using a gyroscopic survey. Taking account of the spatial and temporal variations in the Earth's magnetic field is essential. In addition to this, estimates of the local magnetic field components are used as the primary quality control check for MWD survey data, so their reliability is vital.

There are three basic techniques for determining the wellbore direction when using MWD survey data. Although the terminology varies between directional drilling companies the principles remain the same. Using Halliburton terminology, the first technique is the long collar (LC), where data from all six sensors are used in the calculation of wellbore direction. This method assumes that the magnetometers are positioned in a section of the drill string that is free from any magnetic interference that would otherwise contaminate the measurement of the Earth's magnetic field vector. Although most of the components in a drill string are made of ferrous steel, the MWD tool is placed in a section made from nonmagnetic material and of sufficient length to ensure that the sensors are free from the magnetic interference generated by the rest of the drill string. In this way, the only magnetic error affecting the survey measurements is the result of variations in the direction of the Earth's magnetic field in the horizontal plane, or declination (D). However, as drilling assemblies are increasingly complex, and manufacturing components in non-magnetic material is expensive, it is often difficult to incorporate a sufficient length of non-magnetic housing for the MWD tool. Indeed, there are distinct advantages in placing the MWD tool as close to the drill bit as possible. In particular the time between the application of changes to drilling parameters and measuring the response to them with the MWD tool is reduced.

An alternative technique, which attempts to make use of this advantage, is called short collar (SC) where the MWD tool is placed closer to the drill bit. In this case the sensors are housed in a section of non-magnetic material too short to prevent interference from the rest of the drill string. Within the orthogonal arrangement of magnetometers, one sensor measures along the axis of the drill string whilst the other two measure the magnetic field perpendicular to it. The direction calculated by the SC method assumes that only the axial sensor measurements are corrupted by magnetic interference from the drill string. Estimates of the magnitude of the interference to the axial magnetometer are calculated from values of the total magnetic field strength $(\mathrm{F})$ and dip angle, or magnetic inclination (I), at that location. (Note that magnetic inclination is measured from the horizontal, contrasting with the oil industry convention of measuring wellbore inclination from the vertical). Since the LC assumption of a survey environment free from all forms of magnetic interference is rarely achievable, the SC solution is normally a more accurate calculation of the true wellbore direction.

However, there are also shortcomings with the SC method. Firstly, it relies on having good estimates of absolute values of the Earth's magnetic field at the drilling location, and the algorithms are extremely sensitive to errors in these values at attitudes approaching horizontal and close to the magnetic east-west plane. In the North Sea, variations in the Earth's magnetic field, caused by magnetospheric and ionospheric electric currents and the associated local induced currents, are often sufficient to introduce significant errors if they are not taken into account. Secondly, the SC correction makes the approximation that the errors due to drill string interference are confined to the axial sensor. There are however, several other potential sources of errors including residual calibration errors on one or more of the other five sensors, and other magnetic interference affecting the cross-axial sensors. Reducing the uncertainties in the estimates of the Earth's magnetic field makes it easier to diagnose other sources of error.

Neither LC nor SC solutions will consistently obtain a high degree of accuracy for the wellbore direction and in some instances there are serious weaknesses with both methods. In recent years the industry has developed a third technique called multi-station (MS) analysis. The concept behind MS analysis is that data from a complete series of surveys are used to model the performance of each sensor in the MWD tool. In this method the data are acquired over many rotations of the drill string so, in addition to the SC correction of the axial sensor, it is also possible to analyse the measurements from the cross-axial sensors. MS analysis software operates by predicting theoretical sensor outputs for any given attitude and comparing these with the actual sensor data. The deviations from the predicted model can highlight residual errors in the system helping to distinguish between sensor biases, scale factor errors and misalignments. MS analysis provides a unique magnetic signature for each MWD tool and its drilling assembly. Provided the wellbore geometry and environmental conditions remain constant the correction derived for each assembly can be applied to all the data from the same survey run (Lowdon and Chia, 2003). The form of the magnetic signature makes it possible to determine if the errors are likely to be sensor-related or the result of external environmental factors, which may be an early indication of the onset of a drilling-related issue. The increasing sophistication of MS analysis is heavily dependent on the accuracy of the estimate of the Earth's magnetic field vector. In the North Sea accounting for all sources of the field is normally required to fully differentiate between error sources and to 
calculate the correct borehole direction.

MWD surveys remain the predominant means for controlling the trajectory of directional boreholes and as techniques evolve to further improve the precision of the measurements, the requirement for more accurate estimates of the Earth's magnetic field at the drilling location will continue to increase. In regions such as the North Sea the full benefit of these techniques can only be achieved by accounting for the effects of external field variations.

\section{Estimating the Earth's magnetic field at the drill site}

At any location near the Earth's surface the magnetic field $\boldsymbol{B}$ can be expressed as a vector sum of the contributions from three main sources: the main field generated by the fluid motion in the Earth's core, $\boldsymbol{B}_{\boldsymbol{m}}$; the crustal field from the magnetisation of local rocks, $\boldsymbol{B}_{\boldsymbol{c}}$; and the disturbance field, $\boldsymbol{B}_{\boldsymbol{d}}$, from electrical currents flowing in the ionosphere and magnetosphere:

$\boldsymbol{B}=\boldsymbol{B}_{m}+\boldsymbol{B}_{c}+\boldsymbol{B}_{d}$

Here $\boldsymbol{B}_{\boldsymbol{d}}$ represents both the regular daily, or $\mathrm{Sq}$, variations and irregular magnetic storm variations. $\boldsymbol{B}_{\boldsymbol{m}}$ accounts for approximately $98 \%$ of the field strength at the Earth's surface, and its strength and direction vary slowly with time. In the North Sea the rate of change is typically some tens of $n T$ per year in intensity and a few tenths of a degree per year in direction. In contrast the strength and direction of $\boldsymbol{B}_{\boldsymbol{c}}$ may be regarded as essentially constant at any fixed point, only varying over geological timescales. However, the strength of $\boldsymbol{B}_{\boldsymbol{d}}$, depending mainly on latitude, may vary by hundreds of $\mathrm{nT}$ on timescales of minutes to hours, and it can take any direction, leading to variations in the direction of $\boldsymbol{B}$ of several tenths of a degree during moderate magnetic storms and a few degrees during the most severe storms. During magnetically quiet times, when the $\mathrm{Sq}$ variation dominates $\boldsymbol{B}_{\boldsymbol{d}}$, the fundamental period is $24 \mathrm{~h}$ and its typical range in the North Sea, which varies with geomagnetic latitude, the seasons and the 11-year solar cycle, is a few tens of $\mathrm{nT}$ in $\mathrm{F}$ and approximately $0.2^{\circ}$ in $\mathrm{D}$ and $0.05^{\circ}$ in I. Sq is also dependent on local time, i.e. longitude.

Common practice among drilling surveyors has been to obtain estimates of the field strength and direction at a drilling location by using a spherical harmonic model of the geomagnetic field. The assumption is then made that this is a good estimate of $\boldsymbol{B}$. However, spherical harmonic models of the geomagnetic field are only intended to provide estimates of $\boldsymbol{B}_{\boldsymbol{m}}$. To reduce systematic biases in these geomagnetic field models, contributions from very long wavelength crustal fields and steady components of the disturbance field, may be included in the model. These are generally quite small. Of greater concern is the contribution of the shorter wavelengths of $\boldsymbol{B}_{\boldsymbol{c}}$ and the rapidly varying part of $\boldsymbol{B}_{\boldsymbol{d}}$ as these may be large enough in many parts of the world to cause significant error in the estimate of $\boldsymbol{B}$.
There are, therefore, three magnetic field correction options available to drilling surveyors. One is to correct only for $\boldsymbol{B}_{\boldsymbol{m}}$, which for the reasons stated above will have large errors associated with the estimates. An alternative is to correct for both $\boldsymbol{B}_{\boldsymbol{m}}$ and $\boldsymbol{B}_{\boldsymbol{c}}$. This correction is called In-Field Referencing (IFR) and is commonly used when drilling at lower latitudes, where variations in $\boldsymbol{B}_{\boldsymbol{d}}$ can be regarded as insignificant. In the North Sea and at higher latitudes $\boldsymbol{B}_{\boldsymbol{d}}$ should also be accounted for. By using data from nearby magnetic observatories, estimates of $\boldsymbol{B}_{\boldsymbol{d}}$ can be included to produce the most accurate estimates of $\boldsymbol{B}$ for real-time corrections. This third correction is called Interpolated In-Field Referencing (IIFR). Deriving IIFR data for a particular well is similar to setting up a "virtual" geomagnetic observatory at the drill site.

As well as providing support for existing observatories, the demand from the oil industry for real-time high-quality magnetic observatory data has lead to the establishment of new magnetic observatories in other oil and gas producing areas. Sable Island Observatory, off the coast of Nova Scotia, Canada, became operational in 1999. A magnetic observatory, originally established in 1997 by Halliburton and BGS in Prudhoe Bay, Alaska, was recently upgraded in 2003 and renamed the Jim Carrigan Observatory. Two further observatories have also been established by BGS with the aid of oil industry funds in Ascension Island in 1992 and Port Stanley in the Falkland Islands in 1994. We remark that these observatories not only aid the oil industry but they have also filled gaps in the global network of observatories and have improved the quality and distribution of data for main field modelling and other scientific studies.

\section{The influence of the disturbed field on drilling accuracy}

As discussed in the introduction, for SC and MS, magnetic MWD surveyors require information on both the strength (F) and direction (D and I) of $\boldsymbol{B}$. Knowledge of the errors in the estimates of these three components are also important. At this level accuracies of $0.1^{\circ}$ in $\mathrm{D}, 0.05^{\circ}$ in $\mathrm{I}$ and $50 \mathrm{nT}$ in $\mathrm{F}$ are required (Russell et al., 1995). Turbitt and Clark (1994) demonstrated that data from Lerwick observatory in the United Kingdom could be used to estimate $\boldsymbol{B}_{\boldsymbol{d}}$ throughout the North Sea to within these desired accuracies most of the time. Lerwick data were compared to data from four observatories around the North Sea: Dombås in Norway, Brorfelde in Denmark and Eskdalemuir and Hartland in the UK. The analysis showed that if the user knows $\boldsymbol{B}_{\boldsymbol{m}}, \boldsymbol{B}_{\boldsymbol{c}}$ and $\boldsymbol{B}_{\boldsymbol{d}}$ the confidence level is $99 \%$ over most of the North Sea on a magnetically quiet day. However, during disturbed days the confidence drops to below the $95 \%$ level.

The Turbitt and Clark (1994) study did not consider the errors associated with the estimates of $\boldsymbol{B}_{\boldsymbol{m}}$ and $\boldsymbol{B}_{\boldsymbol{c}}$, however it is clear that the overall error in $\boldsymbol{B}$ can be reduced if $\boldsymbol{B}_{\boldsymbol{d}}$ can be accurately estimated. In order to demonstrate this we compare IIFR $\left(\boldsymbol{B}_{\boldsymbol{m}}+\boldsymbol{B}_{\boldsymbol{c}}+\boldsymbol{B}_{\boldsymbol{d}}\right)$ with IFR $\left(\boldsymbol{B}_{\boldsymbol{m}}+\boldsymbol{B}_{\boldsymbol{c}}\right)$ data. IIFR data are generated using all definitive one-minute values since 
1983, when digital recording at the three UK observatories began, for a hypothetical well situated in the North Sea, at an equivalent latitude to that of Lerwick observatory $\left(60.1^{\circ} \mathrm{N}\right)$. The time period covers two solar activity cycles. The differences represent the geomagnetic disturbance field $\boldsymbol{B}_{\boldsymbol{d}}$ at this site and give a good estimate of the additional error associated with IFR.

The errors associated with the external field by time of day, month and phase of the geomagnetic activity cycle is illustrated in Fig. 1. Three different confidence levels are included. For a Gaussian distribution of errors, the 1-sigma $(\sigma)$ or 1-standard deviation error is equivalent to having $68.3 \%$ confidence that the estimated value is within $\pm \sigma$ of the true value. Additionally, for a Gaussian distribution $2 \sigma$, is equivalent to having $95.4 \%$ confidence and $3 \sigma$ is equivalent to $99.7 \%$ confidence. For any other distribution of errors two or three times $1 \sigma$ is not equivalent to the $95.4 \%$ or $99.7 \%$ confidence levels. As geomagnetic data rarely follow a Gaussian distribution, it is necessary to actually calculate the required equivalent confidence levels. The preferred confidence level in the oil industry for well-planning purposes is that equivalent to $2 \sigma$, i.e. $95.4 \%$.

A number of well-known solar-terrestrial characteristics are identified in these results including the solar activity cycle, the semi-annual effect and local time effects. The significance of the variations differ according to the confidence level used. Considering the local time effects (Fig. 1a), at the $68.3 \%$ confidence level, the Sq variation is most evident, whereas at the $99.7 \%$ level, substorm activity after local midnight and in the early evening is more apparent. At the $95.4 \%$ level of interest to the oil industry these local time variations are a combination of these effects. Examining the seasonal variations (Fig. 1b) the semi-annual effect dominates at the 99.7\% confidence whereas the annual effect due to the seasonal modulation of the $\mathrm{Sq}$ variation is dominant at the $68.3 \%$ level. Again, at the $95.4 \%$ level a combination of both these effects is seen. Figure 1c shows that annually the solar cycle variation is most apparent at the $99.7 \%$ level and also influences the $95.4 \%$ and $68.3 \%$ levels. It should be noted that the external field variation errors at the $95.4 \%$ level frequently exceed the industry specified tolerances of $0.1^{\circ}$ in $\mathrm{D}, 0.05^{\circ}$ in $\mathrm{I}$ and $50 \mathrm{nT}$ in $\mathrm{F}$.

From Fig. 1 it is clear that the errors in the IFR estimates, which are a direct consequence of $\boldsymbol{B}_{\boldsymbol{d}}$, are time dependent. This is well known, as is the fact that these errors vary with magnetic latitude. Figure 2 shows the potential error reduction by using IIFR as opposed to IFR. This is calculated for three hypothetical wells in the North Sea at latitudes similar to that of Hartland observatory $\left(\sim 50^{\circ} \mathrm{N}\right)$, Eskdalemuir observatory $\left(\sim 55^{\circ} \mathrm{N}\right)$ and Lerwick observatory $\left(\sim 60^{\circ} \mathrm{N}\right)$, for three "seasons": winter (November, December, January, February), summer (May, June, July, August) and equinoctial (March, April, September, October). The effect of the different phases of the geomagnetic activity cycle is also considered. The relationship between the geomagnetic and solar activity cycles and how the different phases (maximum, descending, minimum and ascending) of the geomagnetic activity cycle have been determined for this analysis is shown in Fig. 3. The geomagnetic activity cycle has approximately the same 11-year periodicity as the solar activity cycle but with a lag of 2-3 years. As expected, the largest error reduction occurs during the maximum phase of the geomagnetic activity cycle, at the equinox and at the higher latitude well. This analysis also indicates that the reduction in errors by accounting for $\boldsymbol{B}_{\boldsymbol{d}}$ is significant, even at low latitudes during the minimum phase of the cycle. In general by using IIFR the reduction of wellpath uncertainty is of the order of $20 \%$.

To demonstrate the benefit of reducing estimated uncertainties, Fig. 4 shows an example well path with the associated error ellipses for three different survey techniques. The largest ellipses are obtained when MWD is used, correcting only for $\boldsymbol{B}_{\boldsymbol{m}}$, and ignoring $\boldsymbol{B}_{\boldsymbol{c}}$ and $\boldsymbol{B}_{\boldsymbol{d}}$. By accounting for all field sources the associated error ellipses are much reduced. The figure also shows that the gyroscope survey method is expected to provide the smallest positional uncertainty, but MWD with IIFR corrections does not appear to be significantly worse. In addition there are other economic factors in favour of using this technique, as discussed in Sect. 1.

\section{The application of geomagnetic data in the oil industry}

Using IIFR to account for the external field has many advantages for the oil industry. Prior to the introduction of IIFR, in high latitude areas such as Alaska the variations in the disturbance field could be so great during magnetic storms, magnetic MWD surveying was impossible. Entire sections of a borehole would have to be re-surveyed once the magnetic activity had subsided. In the North Sea the disturbance field variation is less extreme, particularly at UK latitudes. But, it is still sufficient to cause major problems when using magnetic MWD data for precise control of the position of a borehole, particularly under rapid drilling conditions. The principal value of using the measured disturbance field in the North Sea lies in the improvement in the accuracy of the mathematical methods now used to enhance conventional MWD data. As described in the introduction these mathematical techniques rely heavily on estimates of the local $\mathrm{F}$ and I values. At the latitude of the North Sea small errors in these estimates can manifest themselves as disproportionately large errors in the calculated wellbore direction.

As well as the reduction in error, the provision of realtime geomagnetic data provides independent validation of the outputs of downhole MWD survey tools. For example, some operators require two independent MWD survey tools to be used to validate the wellbore survey. Using one MWD survey tool and IIFR is now recognised as fulfilling this two-tool requirement. Good correlation between the MWD and IIFR results show that downhole instruments are performing correctly, whereas a miscorrelation can highlight a problem with the MWD survey. Figure 5 shows MWD survey results for a high-latitude North Sea well in comparison with IIFR data derived from observatory data. The 
3-sigma equivalent $(99.7 \%) \quad$ 2-sigma equivalent $(95.4 \%) \quad$ 1-sigma equivalent $(68.3 \%)$
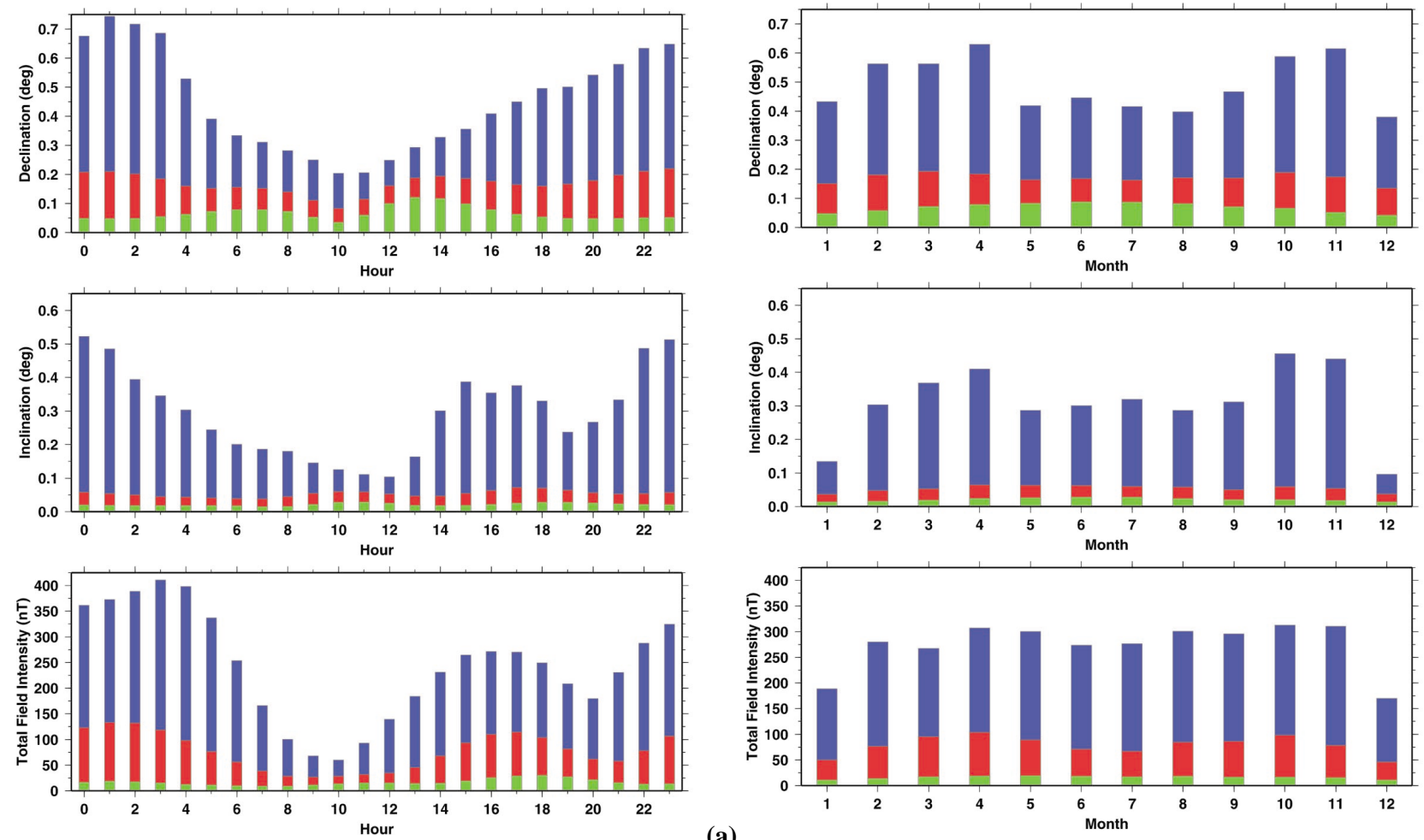

(a)

3-sigma equivalent (99.7\%) 2-sigma equivalent (95.4\%) $\quad$ 1-sigma equivalent (68.3\%)
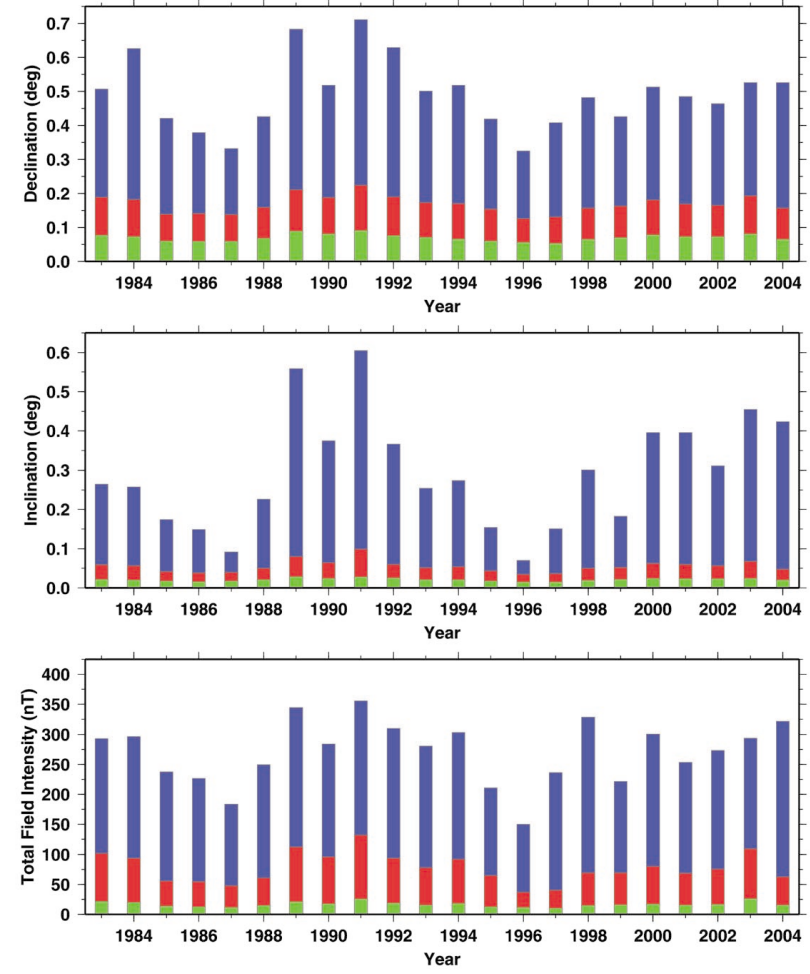

(c)

Fig. 1. Three histograms showing errors associated with the external field by hour (a), showing local time variations; by month (b), showing seasonal variations; and by year (c), showing solar cycle variations. For each, three confidence levels are shown: $1 \sigma$ equivalent $(68.3 \%)$ in green, $2 \sigma$ equivalent $(95.4 \%)$ in red and $3 \sigma$ equivalent $(99.7 \%)$ in blue. 

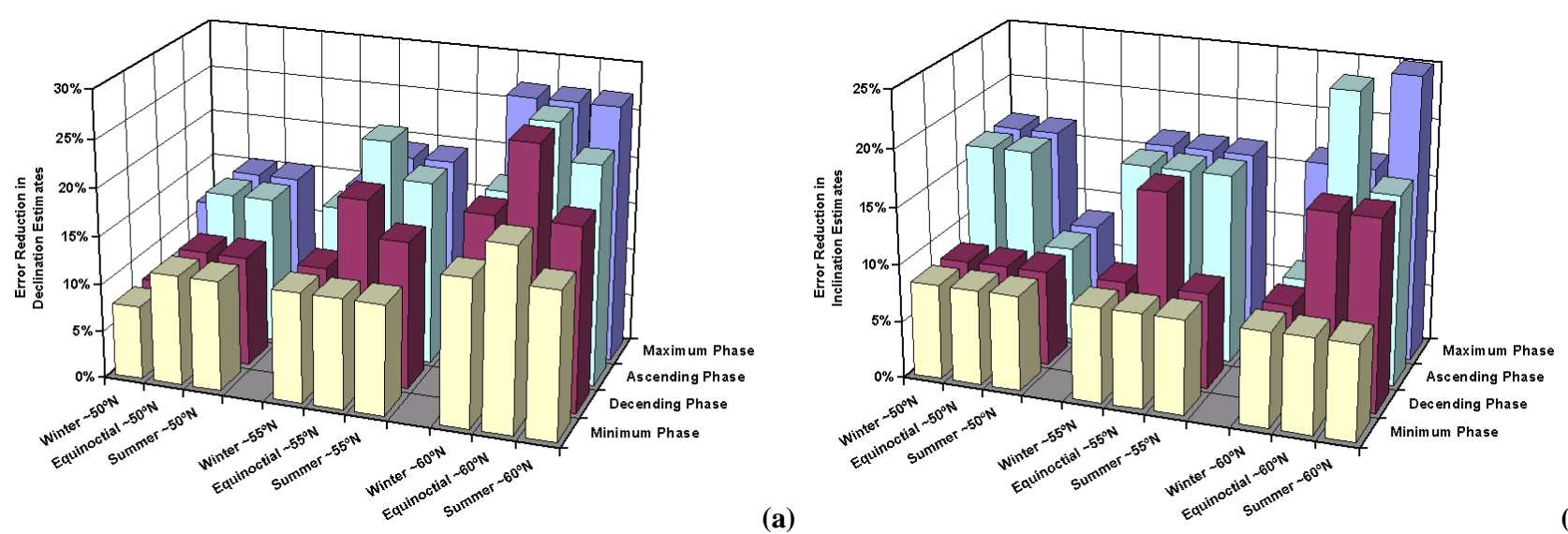

(b)

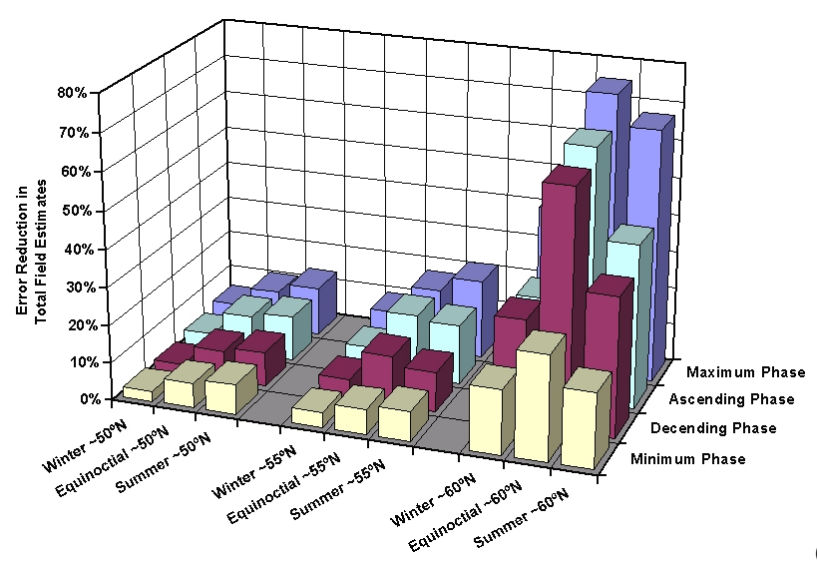

(c)

Fig. 2. The reduction in errors, as a percentage, in the estimates for declination (a), inclination (b) and total field intensity (c), when accounting for $\boldsymbol{B}_{\boldsymbol{d}}$ as well as $\boldsymbol{B}_{\boldsymbol{m}}+\boldsymbol{B}_{\boldsymbol{c}}$. The results shown are those obtained for the error reduction over 22 years (1983 to 2004) as a function of geomagnetic activity cycle (four phases as described in Fig. 3), latitude (three locations at approximately $50^{\circ}, 55^{\circ}$ and $60^{\circ} \mathrm{N}$ ) and season (winter, summer and equinoctial).

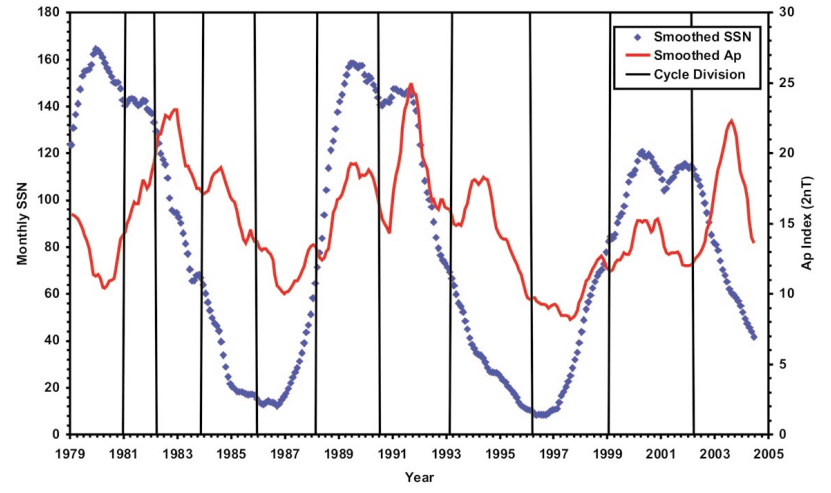

Fig. 3. The solar and geomagnetic activity cycle as represented by smoothed sunspot number (SSN) and smoothed planetary activity index Ap respectively. The division of the geomagnetic activity cycle into four phases (minimum, ascending, maximum and descending) is indicated by vertical lines. close correlation between the IIFR data and the MWD data recorded downhole give confidence that the MWD tool is working correctly. In this figure the correlation is especially good between 2.3-2.5 km when the Earth's magnetic field was changing rapidly.

There can be many sources of error in MWD surveys: the tool may not work correctly; a bad measurement might be taken due to downhole vibration or from the effect of magnetically susceptible drilling fluid (magnetic mud). In the Norwegian sector it is very common to use recycled oil-based mud as a drilling fluid. Over many months this same mud is used and re-used causing large quantities of abraded steel to become suspended in the fluid. This has the effect of contaminating the MWD sensor readings as the steel particles shield the tool from the full effects of the Earth's magnetic field (Wilson and Brooks, 2001; Torkildsen et al., 2004). If present, this source of error can dominate most other error sources. It mostly affects the two cross-axial MWD sensors. MS analysis software can correct a wellbore direction for the effect of interference caused by magnetic mud but to do so 


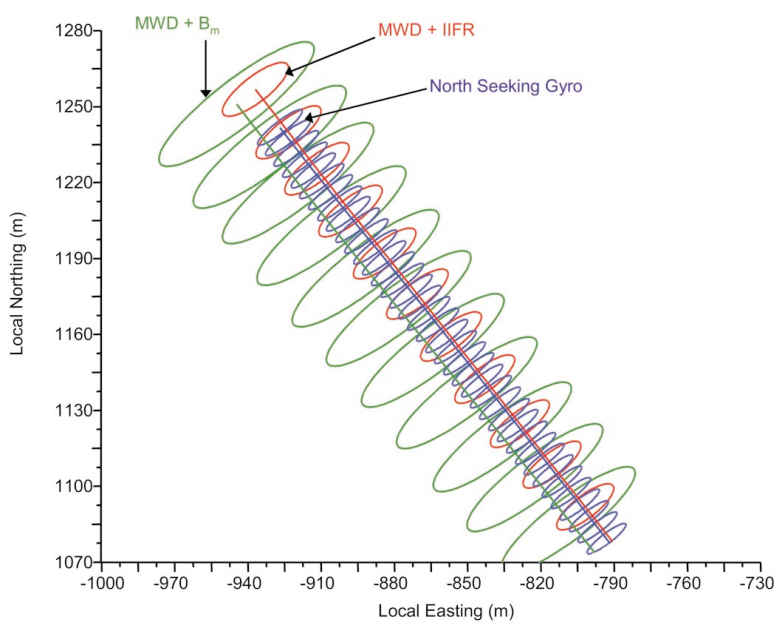

Fig. 4. Three sets of error ellipses on a section of wellpath demonstrating the size of errors associated with two directional survey correction methods for magnetic MWD surveys and a gyroscopic survey. Those associated with MWD and main field $\left(\boldsymbol{B}_{\boldsymbol{m}}\right)$ corrections only are shown in green, those for MWD with IIFR in red, and those derived for a north-seeking gyroscope are shown in blue. The data are from a 4-day period in 2001 .

the local geomagnetic field must be well known. Figure 6 demonstrates the effect magnetic mud can have on drilling azimuth. Since cross-axial sensors are more affected, the correction is significantly different from that of the SC correction. In this example it resulted in the azimuth error averaging nearly $3^{\circ}$. The MS correction could only be accurately derived because the residual uncertainty of the field had been reduced to insignificant levels by application of the IIFR correction. Without IIFR, MS techniques would be unable to differentiate with sufficient confidence between the drill string interference error and the effect of the magnetic mud.

One of the main applications of MWD is in horizontal drilling, where the wellbore inclination is greater than $85^{\circ}$. This technology has allowed drillers to source reservoirs that would otherwise be inaccessible or uneconomic to drill. When drilling wells that are close to horizontal and within $20^{\circ}$ of magnetic east-west, the drill string correction algorithms are very sensitive to small errors in the magnetic field values used. Figure 7 shows an example horizontal well path with a small $( \pm 10 \mathrm{~m})$ lateral target. The green line shows how positional uncertainty could cause a gradual deviation in the well path from the planned trajectory resulting in the target being missed. The improvement gained by including the disturbance field, $\boldsymbol{B}_{\boldsymbol{d}}$, can help avoid this situation.

Full correction of wellbore directions recorded using MWD can help reduce non-productive time on the rig. Gyroscopic surveys on the other hand can be very time consuming. Also, with several oil wells being drilled from one single platform or subsea template, close approaches to other wells are possible. This can often result in the adjacent wells being temporarily closed off (shut-in) for safety reasons. By

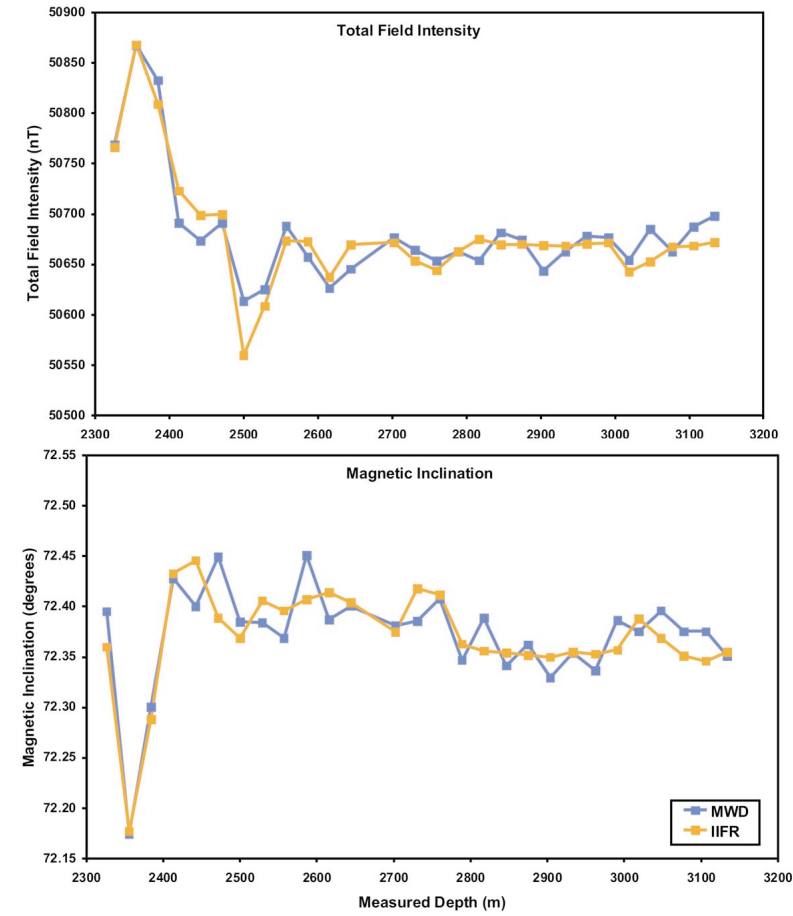

Fig. 5. A comparison of magnetic field values measured by the MWD survey tools (blue) with those calculated by IIFR (orange). Total field intensity is shown at the top and magnetic inclination at the bottom. This survey spans a period of 8 days during 2001 .

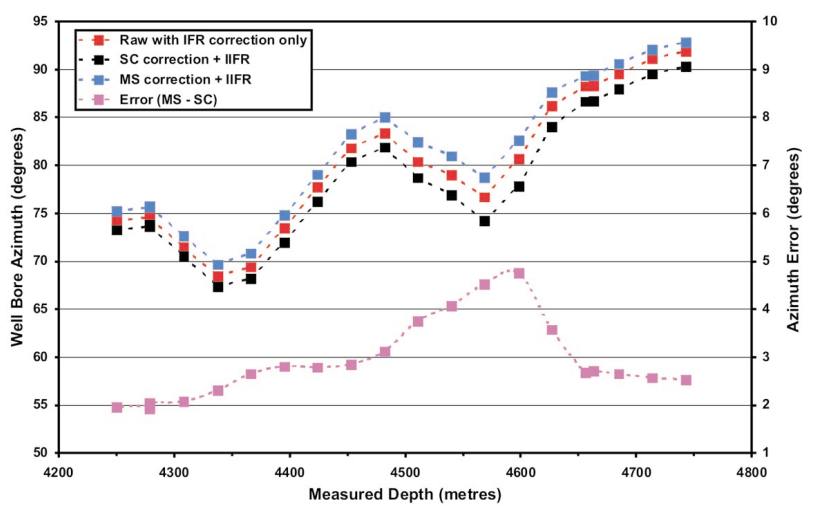

Fig. 6. A comparison of MWD survey correction methods showing the wellbore azimuth, over 2 days of drilling, at regular intervals along the well path with respect to the measured depth. The red trace is the raw measured azimuth without any corrections for magnetic interference near the sensors (i.e. equivalent to LC with IFR correction only). The black trace shows the additional SC correction that would be applied assuming all the magnetic interference was due to axial drill string interference. The blue trace is the final MS corrected azimuth which includes a correction of the cross-axial sensor data for other interference including magnetically susceptible drilling fluid. Also shown is the difference between SC and MS in sense of MS-SC (lower pink trace). This indicates the azimuth error, as shown on the right hand y-axis, that would have been incurred had the MS method not been used. 


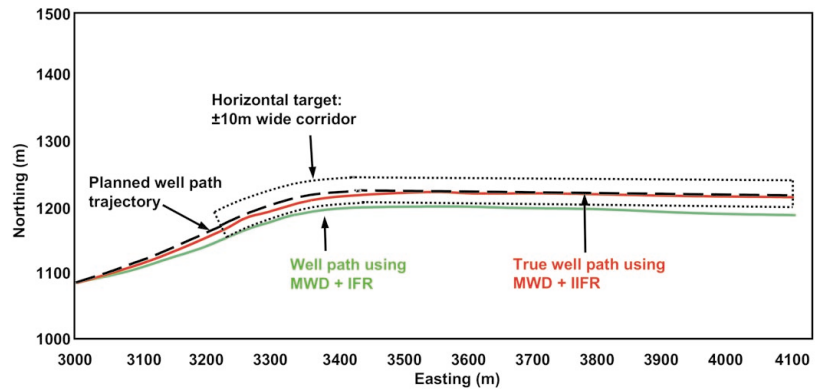

Fig. 7. A horizontal well path drilled towards magnetic east demonstrating the deviation outside a $\pm 10 \mathrm{~m}$ wide corridor from the planned path (dashed line) when MWD with IFR corrections is used (green line). The true well path position (red line) is derived from MWD and MS analysis after correcting for all field sources with IIFR. This wellpath was drilled over 6 days in 2004

accounting for all sources of the Earth's magnetic field the improved accuracy in the estimates and subsequent reduced uncertainty in azimuth errors reduces the need to shut-in the other wellbores, thereby helping to avoid lost production time.

\section{Conclusions}

In this paper we have highlighted the importance of external magnetic field variations and the effects of space weather on drilling operations in the North Sea. We have shown how the well known climatological variations in geomagnetic activity, such as the semi-annual, solar cycle and the local time preference for substorm activity, affects the errors associated with drilling accuracy, if the external field variations are not accounted for in corrections to MWD surveys. We have discussed some of the techniques drilling surveyors use to determine positional accuracy and the benefits of using fully corrected MWD surveys.

Comparison of IFR corrections $\left(\boldsymbol{B}_{\boldsymbol{m}}+\boldsymbol{B}_{c}\right)$ with IIFR corrections $\left(\boldsymbol{B}_{\boldsymbol{m}}+\boldsymbol{B}_{\boldsymbol{c}}+\boldsymbol{B}_{\boldsymbol{d}}\right)$ over 22 years shows that the expected reduction in wellpath uncertainty by using IIFR is of the order of $20 \%$. We therefore conclude that if high quality data from a nearby magnetic observatory are available then it is always better to use these to correct MWD surveys at drilling sites at magnetic latitudes similar to or greater than that of the North Sea.
Acknowledgements. This paper is published with the permission of the Executive Director of the British Geological Survey. The authors would like to thank D. McRobbie and S. McCulloch of Halliburton for valuable discussions and D. Kerridge and A. Thomson of BGS for their reviews and advice on the paper. We would also like to acknowledge the work of ESA/ESTEC and the COST 724 Action on Space Weather that provided the opportunity for this work.

Topical Editor M. Pinnock thanks J. Watermann and another referee for their help in evaluating this paper.

\section{References}

Lowdon, R. M and Chia C. R.: Multistation analysis and geomagnetic referencing significantly improve magnetic survey results, Society of Petroleum Engineers, available from: http://www.spe.org/elibinfo/eLibrary_Papers/spe/2003/ 03DC/00079820/00079820.htm, Technical Paper SPE/IADC $79820,2003$.

Russell, J. P., Shiells, G., and Kerridge, D. J.: Reduction of wellbore positional uncertainty through application of a new geomagnetic in-field referencing technique, Society of Petroleum Engineers, available from: http://www.spe.org/elibinfo/eLibrary Papers/spe/1995/95SPE/00030452/00030452.htm, Technical Paper SPE 30452, 1995.

Torkildsen, T., Edvardsen, I., Fjogstad, A., Saasen, A., Amundsen, P., and Omland T.: Drilling fluid affects MWD magnetic azimuth and wellbore position, Society of Petroleum Engineers, available from: http://www.spe.org/elibinfo/eLibrary Papers/spe/2004/04DC/00087169/00087169.htm, Technical Paper SPE 87169, 2004.

Turbitt, C. W. and Clark, T. D. G.: The Use of Lerwick variometer measurements to estimate magnetic disturbances over the North Sea, British Geological Survey, Technical Report, WM/94/21C, 1994.

Wilson, H. and Brooks, A. G.: Wellbore position errors caused by drilling-fluid contamination, SPE Drill. C., 16, 208-213, 2001. 